\title{
OBTAINING UPPER BOUNDS OF HEAT KERNELS FROM LOWER BOUNDS
}

\author{
ALEXANDER GRIGOR'YAN, JIAXIN HU, AND KA-SING LAU
}

\begin{abstract}
We show that a near-diagonal lower bound of the heat kernel of a Dirichlet form on a metric measure space with a regular measure implies an on-diagonal upper bound. If in addition the Dirichlet form is local and regular then we obtain a full off-diagonal upper bound of the heat kernel provided the Dirichlet heat kernel on any ball satisfies a near-diagonal lower estimate. This reveals a new phenomenon in the relationship between the lower and upper bounds of the heat kernel.
\end{abstract}

\section{Contents}

1. Introduction 1

2. Preliminaries 3

3. Near-diagonal lower estimates imply on-diagonal upper bounds 5

4. Local lower estimates imply full upper bounds 9

$\begin{array}{lr}\text { References } & 16\end{array}$

\section{INTRODUCTION}

There has been a vast literature on two-sided estimates of heat kernels on Riemannian manifolds, infinite graphs, fractals, and, more generally, on metric measure spaces. The reader may consult $[8,10,14,29,30]$ for Riemannian manifolds, $[5,9,19,20]$ for infinite graphs, $[2,3,25]$ for fractals or metric spaces, and the references therein.

In a majority of the proofs of two-sided estimates for the heat kernel, one normally obtains first the upper bound and then use it in order to prove the lower bound. This method goes back to the pioneering work by Aronson [1] and since that time has become standard in the heat kernel literature (see, for example, [2, 11, 22, 28, 31]).

Our purpose in this paper is to show that, conversely, certain heat kernel lower bounds imply the upper bounds! As far as we know, this is the first result of this kind.

Let $(M, d, \mu)$ be a metric measure space endowed with a Dirichlet form $(\mathcal{E}, \mathcal{F})$ in $L^{2}(M, \mu)$. The main examples of such a space are as follows:

(1) $M$ is a Riemannian manifold, $d$ is the geodesic distance, $\mu$ is the Riemannian measure, and $\mathcal{E}$ is the classical Dirichlet form

$$
\mathcal{E}(f)=\int_{M}|\nabla f|^{2} d \mu
$$

with domain $\mathcal{F}=W_{0}^{1}(M)$.

Date: June 28, 2006.

AG was supported by a Royal Society Kan Tong Po Visiting Professorship in CUHK and by a Visiting Professorship of IMS CUHK. JH was supported by NSFC (Grant No. 10371062), HKRGC Grant and a visiting grant from Universität Bielefeld. KSL was partially supported by the HKRGC Grant in CUHK. 
(2) $M$ is a fractal subset of $\mathbb{R}^{N}$ such as the Sierpinski gasket or the Sierpinski carpet. In this case, $d$ is usually the extrinsic distance from $\mathbb{R}^{N}$ and $\mu$ is the Hausdorff measure of a proper dimension. The definition of a Dirichlet form is highly nontrivial. For a certain class of fractals, one first defines a discrete Dirichlet form on a graph approximation of $M$ and then takes a properly scaled limit.

Assume that the heat semigroup associated with $(\mathcal{E}, \mathcal{F})$ has an integral kernel, which is then called the heat kernel of $(\mathcal{E}, \mathcal{F})$ and is denoted by $p_{t}(x, y)$. In general, this function is measurable with respect to $x, y$ for any $t>0$. For the sake of Introduction, assume in addition that $p_{t}(x, y)$ is continuous in $x, y$. Note that if $M$ is a Riemannian manifold then $p_{t}(x, y)$ is the minimal positive fundamental solution to the heat equation on $M$.

Let measure $\mu$ be $\alpha$-regular, that is, for any metric ball $B(x, r)$,

$$
\mu(B(x, r)) \asymp r^{\alpha} .
$$

Our first result (Theorem 3.3) says that if the heat kernel satisfies the near-diagonal lower estimate

$$
p_{t}(x, y) \geq c t^{-\alpha / \beta} \text { for all } x, y \in M \text { such that } d(x, y) \leq \delta t^{1 / \beta}
$$

where $\beta, c, \delta$ are positive constants, then it satisfies also the on-diagonal upper estimate

$$
p_{t}(x, x) \leq C t^{-\alpha / \beta} \text { for all } x \in M, t>0 .
$$

The proof of this result is based on the following two components:

(1) We introduce a family $W^{\beta / 2,2}$ of Besov function spaces on the metric measure space $(M, d, \mu)$ and prove the Nash type inequality for the norm in these spaces (see Proposition 2.1 below). This component requires only the regularity of the measure.

(2) Using (1.1), we obtain the embedding estimate (3.6), which implies the Nash inequality for the Dirichlet form $\mathcal{E}$. Then (1.2) follows by the Nash argument [27].

The hypothesis that $\mu$ is $\alpha$-regular is essential. We give an example showing that if this hypothesis fails, then the near-diagonal lower estimate (1.1) does not imply (1.2) (see Example 3.7).

A natural question arises whether one can obtain in the same setting also an off-diagonal upper bound for $p_{t}(x, y)$ showing the decay as $d(x, y) \rightarrow \infty$. For that, assume in addition that the Dirichlet form $(\mathcal{E}, \mathcal{F})$ is regular and local. Our conjecture is that, under the above hypotheses, (1.1) implies the following full upper bound:

$$
p_{t}(x, y) \leq \frac{C}{t^{\alpha / \beta}} \exp \left(-\left(\frac{d^{\beta}(x, y)}{C^{\prime} t}\right)^{\frac{1}{\beta-1}}\right) .
$$

Note that (1.3) and a matching lower bound for $p_{t}(x, y)$ are known to be true for a large class of fractal sets, which are then characterized by the two parameters $\alpha$ and $\beta$ (in fact, $\alpha$ is the Hausdorff dimension of $M$ and $\beta$ is a so called walk dimension).

In the case when $M$ is a Riemannian manifold and $\beta=2$, this conjecture is true because (1.3) follows from (1.2) alone (Corollary 3.5). In the general case, we have been able to prove the following two weaker versions of this conjecture.

(1) A somewhat stronger condition than (1.1), called the local lower estimate of the heat kernel (see (LLE) in Section 4) does imply (1.3) (Theorem 4.2).

(2) If a near-diagonal lower bound (1.1) holds together with the following time-independent upper bound

$$
p_{t}(x, y) \leq C d(x, y)^{-\alpha},
$$

then (1.3) is true (Theorem 4.6). 
The locality of the form $(\mathcal{E}, \mathcal{F})$ is necessary for $(1.3)$, which is shown in Example 4.7.

Our results are new even for the case of Riemannian manifolds with $\beta=2$. For example, Theorem 4.2 provides the following new proof of the fact that Moser's parabolic Harnack inequality (see [26]) for the heat equation on a manifold with $\alpha$-regular measure implies the heat kernel two sided Gaussian estimates (see [22] for another proof of this result). Indeed, the condition (LLE) mentioned above is an analogue of (1.1) for the heat kernel $p_{t}^{B}$ in a ball $B \subset M$ with the Dirichlet boundary condition, which is somewhat stronger than (1.1). By the classical Aronson argument [1], the parabolic Harnack inequality implies (LLE) with $\beta=2$. By Theorem 4.2, we obtain the upper bound (1.3). The matching lower bound follows from (1.1) again by Aronson's chain argument.

Notation. Letters $c, c^{\prime}, c_{0}, C$ etc. denote positive constants, whose values may change at each occurrence. If $f$ and $g$ are two non-negative functions then we write $f \asymp g$ if, for some $C>0$,

in the common domain of $f$ and $g$.

$$
C^{-1} g \leq f \leq C g
$$

\section{Preliminaries}

Let $(M, d)$ be a locally compact, separable metric space, and let $\mu$ be a Radon measure supported on $M$. For $1 \leq p \leq \infty$, denote by $L^{p}:=L^{p}(M, \mu)$ the usual space of all $p$-integrable real-valued functions on $M$ with the norm

$$
\|f\|_{p}=\left(\int_{M}|f(x)|^{p} d \mu(x)\right)^{1 / p}
$$

(with the obvious modification if $p=\infty$ ).

Let $(\mathcal{E}, \mathcal{F})$ be a Dirichlet form on $L^{2}(M, \mu)$. In the sequel we use the convenient abbreviation $\mathcal{E}(f):=\mathcal{E}(f, f)$ for $f \in \mathcal{F}$. Let $H$ be the generator of $(\mathcal{E}, \mathcal{F})$, that is, $H$ is a non-negative definite self-adjoint operator in $L^{2}$ with domain $\operatorname{dom}(H) \subset \mathcal{F}$, and

$$
(H f, g)=\mathcal{E}(f, g) \quad(f \in \operatorname{dom}(H), g \in \mathcal{F}),
$$

where $($,$) is the inner product on L^{2}$. The generator $H$ gives rise to the semigroup

$$
T_{t}=e^{-t H} \quad(t \geq 0)
$$

which is a family of bounded self-adjoint operator in $L^{2}$. In addition, the semigroup $\left\{T_{t}\right\}$ is Markovian, that is, if $0 \leq f \leq 1$ a.e., then

$$
0 \leq T_{t} f \leq 1
$$

a.e. for all $t \geq 0$, see [12, Theorem 1.4.1, p. 23]. A family $\left\{p_{t}(x, y)\right\}_{t>0}$ of measurable functions on $M \times M$ is termed the heat kernel of the form $(\mathcal{E}, \mathcal{F})$ if $p_{t}(x, y)$ is an integral kernel of $T_{t}$, that is

$$
T_{t} f(x)=\int_{M} p_{t}(x, y) f(y) d \mu(y) \quad \text { for a.e. } x \in M
$$

for all $t>0$ and $f \in L^{2}$.

For $x \in M$ and $r>0$, let $B(x, r)=\{y \in M: d(y, x)<r\}$ be the open ball in $M$. Fix some $r_{0} \in(0, \infty]$ throughout this paper. For $\alpha>0$, we say that $\mu$ is lower $\alpha$-regular if there exists a constant $c_{1}>0$ such that

$$
\mu(B(x, r)) \geq c_{1} r^{\alpha}
$$

for $\mu$-almost all $x \in X$ and $0<r<r_{0}$, and $\mu$ is upper $\alpha$-regular if there exists a constant $c_{2}>0$ such that

$$
\mu(B(x, r)) \leq c_{2} r^{\alpha}
$$


for $\mu$-almost all $x \in X$ and $0<r<r_{0}$. We say that $\mu$ is $\alpha$-regular if $\mu$ is both upper and lower $\alpha$-regular.

For any $\sigma>0$, define a non-negative functional $W_{\sigma}(f)$ on $L^{2}$ by

$$
W_{\sigma}(f):=\sup _{0<r<r_{0}} r^{-2 \sigma} \int_{M}\left[\frac{1}{\mu(B(x, r))} \int_{B(x, r)}|f(y)-f(x)|^{2} d \mu(y)\right] d \mu(x) .
$$

In particular, if $\mu$ is $\alpha$-regular, then for any $\beta>0$,

$$
W_{\beta / 2}(f) \asymp \sup _{0<r<r_{0}} r^{-\alpha-\beta} \int_{M}\left[\int_{B(x, r)}|f(y)-f(x)|^{2} d \mu(y)\right] d \mu(x) .
$$

Define the Banach space $W^{\sigma, 2}$ by

$$
W^{\sigma, 2}=W^{\sigma, 2}(M, d, \mu):=\left\{f \in L^{2}: W_{\sigma}(f)<\infty\right\} .
$$

with the norm

$$
\left(\|f\|_{2}^{2}+W_{\sigma}(f)\right)^{1 / 2}
$$

The space $W^{\sigma, 2}$ admits the following Nash inequality.

Proposition 2.1. Assume that $\mu$ is $\alpha$-regular and $\beta>0$. Then, for all $f \in W^{\beta / 2,2}$,

$$
\|f\|_{2}^{2\left(1+\frac{\beta}{\alpha}\right)} \leq c\left(r_{0}^{-1}\|f\|_{2}^{2}+W_{\beta / 2}(f)\right)\|f\|_{1}^{\frac{2 \beta}{\alpha}},
$$

where $c>0$ depends only on $\alpha, \beta, c_{1}, c_{2}$.

Proof. We can assume that $f \in L^{1} \cap W^{\beta / 2,2}$. For any such $f$ and $r>0$, set

$$
f_{r}(x):=\frac{1}{\mu(B(x, r))} \int_{B(x, r)} f(y) d \mu(y) .
$$

In the remainder of the proof, we denote by $c$ a positive constant depending only on $\alpha, \beta, c_{1}, c_{2}$ but whose value may be changed at each instance. Note that

$$
\left\|f_{r}\right\|_{1} \leq c\|f\|_{1} .
$$

By (2.4), we see that

$$
\left\|f_{r}\right\|_{\infty} \leq c_{1}^{-1} r^{-\alpha}\|f\|_{1}
$$

for $0<r<r_{0}$. Combining (2.10) and (2.9), we obtain that

$$
\left\|f_{r}\right\|_{2}^{2} \leq\left\|f_{r}\right\|_{\infty}\left\|f_{r}\right\|_{1} \leq c r^{-\alpha}\|f\|_{1}^{2} .
$$

On the other hand, using the Cauchy-Schwarz inequality, we have that

$$
\begin{aligned}
\left\|f_{r}-f\right\|_{2}^{2} & =\int_{M}\left(\frac{1}{\mu(B(x, r))} \int_{B(x, r)}(f(y)-f(x)) d \mu(y)\right)^{2} d \mu(x) \\
& \leq \int_{M}\left(\frac{1}{\mu(B(x, r))} \int_{B(x, r)}(f(y)-f(x))^{2} d \mu(y)\right) d \mu(x) \\
& =r^{\beta}\left\{r^{-\beta} \int_{M}\left(\frac{1}{\mu(B(x, r))} \int_{B(x, r)}(f(y)-f(x))^{2} d \mu(y)\right) d \mu(x)\right\} \\
& \leq r^{\beta} W_{\beta / 2}(f) .
\end{aligned}
$$

Therefore, it follows from (2.11) and (2.12) that

$$
\|f\|_{2}^{2} \leq 2\left(\left\|f_{r}\right\|_{2}^{2}+\left\|f_{r}-f\right\|_{2}^{2}\right) \leq c\left(r^{-\alpha}\|f\|_{1}^{2}+r^{\beta} W_{\beta / 2}(f)\right),
$$


for $0<r<r_{0}$. Clearly, if $r \geq r_{0}\left(\right.$ and $\left.r_{0}<\infty\right)$, then we have that

$$
\|f\|_{2}^{2} \leq\left(\frac{r}{r_{0}}\right)^{\beta}\|f\|_{2}^{2}
$$

which together with (2.13) yields that

$$
\|f\|_{2}^{2} \leq c\left(r^{-\alpha}\|f\|_{1}^{2}+r^{\beta}\left(r_{0}^{-1}\|f\|_{2}^{2}+W_{\beta / 2}(f)\right)\right)
$$

for all $r>0$. If $f \equiv 0$, then (2.8) is trivial. Otherwise

$$
r_{0}{ }^{-1}\|f\|_{2}^{2}+W_{\beta / 2}(f) \neq 0 .
$$

Indeed, if this expression vanishes, then we would have that $r_{0}=\infty$ and $f=$ const $\neq 0$. Since $\mu$ is $\alpha$-regular, it follows from $r_{0}=\infty$ that $\mu(M)=\infty$, and so $f=$ const $\notin L^{2}$. This is a contradiction. Hence, letting

$$
r=\left(\frac{\|f\|_{1}^{2}}{r_{0}^{-1}\|f\|_{2}^{2}+W_{\beta / 2}(f)}\right)^{1 /(\alpha+\beta)}
$$

in (2.14), we obtain (2.8).

The proof of Proposition 2.1 is motivated by [23, Theorem 3.1].

\section{NEAR-DiAgONAL LOWER ESTIMATES IMPLY ON-DIAGONAL UPPER BOUNDS}

Assume that the Dirichlet form $(\mathcal{E}, \mathcal{F})$ possesses a heat kernel $p_{t}(x, y)$. We say that $p_{t}(x, y)$ satisfies a near-diagonal lower estimate if, for some $\delta, c_{0}>0, \beta>1$,

$$
p_{t}(x, y) \geq c_{0} t^{-\alpha / \beta}
$$

for all $0<t<\delta r_{0}^{\beta}$ and $\mu$-almost all $x, y \in M$ satisfying

$$
d(x, y)<\delta t^{1 / \beta},
$$

where $\alpha$ is the same as in (2.4). Under a certain additional assumption on the metric $d$, for example the chain condition, (NLE) allows us to obtain a full lower estimate for $p_{t}(x, y)$ for all $0<t<r_{0}^{\beta}$ and $\mu$-almost all $x, y \in M$. We say that $(M, d)$ satisfies the chain condition if, for any distinct points $x, y \in X$ and any integer $n \geq 1$, there exist a constant $c>0$ and a sequence of points $\left\{x_{k}\right\}_{k=0}^{n}$ in $X$ such that $x_{0}=x, x_{n}=y$, and

$$
d\left(x_{i}, x_{i+1}\right) \leq c n^{-1} d(x, y) \quad(0 \leq i \leq n-1) .
$$

For instance, the chain condition is satisfied if $(M, d)$ is a geodesic space.

Proposition 3.1. Let $(M, d)$ be a metric space satisfying the chain condition, and let $\mu$ be lower $\alpha$-regular. Assume that the heat kernel $p_{t}(x, y)$ of the Dirichlet form $(\mathcal{E}, \mathcal{F})$ exists and satisfies $(N L E)$. Then $p_{t}(x, y)$ satisfies the off-diagonal lower bound

$$
p_{t}(x, y) \geq c t^{-\alpha / \beta} \exp \left(-c^{\prime}\left(\frac{d(x, y)}{t^{1 / \beta}}\right)^{\frac{\beta}{\beta-1}}\right)
$$

for all $0<t<r_{0}^{\beta}$ and $\mu$-almost all $x, y \in M$, for some $c, c^{\prime}>0$.

See [2] or [17, Corollary 3.5] for the proof.

The following is the key result in this paper, showing that (NLE) implies the Nash inequality for $(\mathcal{E}, \mathcal{F})$. 
Theorem 3.2. Let $(M, d)$ be a metric space with a lower $\alpha$-regular measure $\mu$. Assume that the heat kernel of the Dirichlet form $(\mathcal{E}, \mathcal{F})$ exists and satisfies $(N L E)$. Then, for all $f \in \mathcal{F}$,

$$
\|f\|_{2}^{2\left(1+\frac{\beta}{\alpha}\right)} \leq c\left(r_{0}^{-1}\|f\|_{2}^{2}+\mathcal{E}(f)\right)\|f\|_{1}^{\frac{2 \beta}{\alpha}}
$$

where $c$ (independent of $r_{0}$ ) depends only on the constants from the hypothesis.

Proof. First note that (NLE) implies that the measure $\mu$ is upper $\alpha$-regular, see $[17,(3.3)$, p.2071] for the case $r_{0}=\infty$. For $r_{0}<\infty$, the proof is the same. Indeed, fix a ball $B\left(x_{0}, r\right)$ with $0<r \leq \varepsilon r_{0}$ where

$$
\varepsilon=\frac{1}{2} \delta^{1+1 / \beta}
$$

with the same $\delta$ as in (NLE). Let $f=\mathbf{1}_{B\left(x_{0}, r\right)}$. Then, for any $t>0$ and almost all $x \in B\left(x_{0}, r\right)$,

$$
1 \geq T_{t} f(x)=\int_{B\left(x_{0}, r\right)} p_{t}(x, y) d \mu(y) \geq \mu\left(B\left(x_{0}, r\right)\right) \underset{y \in B\left(x_{0}, r\right)}{\operatorname{essinf}} p_{t}(x, y),
$$

whence

$$
\mu\left(B\left(x_{0}, r\right)\right) \leq\left(\underset{x, y \in B\left(x_{0}, r\right)}{\operatorname{essinf}} p_{t}(x, y)\right)^{-1}
$$

Choosing $t$ such that

$$
\delta t^{1 / \beta}=2 r
$$

we see that, for all $x, y \in B\left(x_{0}, r\right)$,

$$
d(x, y)<2 r=\delta t^{1 / \beta},
$$

and

$$
t=(2 r / \delta)^{\beta} \leq\left(2 \varepsilon r_{0} / \delta\right)^{\beta}=\delta r_{0}^{\beta} .
$$

Therefore, (NLE) implies that

$$
\underset{x, y \in B\left(x_{0}, r\right)}{\operatorname{essinf}} p_{t}(x, y) \geq c_{0} t^{-\alpha / \beta}=c r^{-\alpha},
$$

whence $\mu$ is upper $\alpha$-regular for $0<r \leq \varepsilon r_{0}$ by virtue of (3.3). Now, if $r_{0}<\infty$ and $\varepsilon r_{0}<r<r_{0}$, then

$$
\mu\left(B\left(x_{0}, r\right)\right) \geq \mu\left(B\left(x_{0}, \varepsilon r_{0}\right)\right) \geq c\left(\varepsilon r_{0}\right)^{\alpha}=c^{\prime} r^{\alpha},
$$

and so $\mu$ is upper $\alpha$-regular for all $0<r<r_{0}$ by adjusting the constant.

For any $f \in L^{2}$, set

$$
\mathcal{E}_{t}(f)=\frac{1}{t} \int_{M}\left(f-T_{t} f\right) f d \mu .
$$

By [12, Lemma 1.3.4, p.22], the family $\left\{\mathcal{E}_{t}(f)\right\}$ increases as $t \downarrow 0$ and tends to $\mathcal{E}(f)$, for any $f \in \mathcal{F}$. Using this and (2.2), we obtain that, for any $t>0$ and $r>0$,

$$
\begin{aligned}
\mathcal{E}(f) \geq & \frac{1}{t} \int_{M}\left(f(x)-T_{t} f(x)\right) f(x) d \mu(x) \\
= & \frac{1}{2 t}\left\{\int_{M} \int_{M}(f(x)-f(y))^{2} p_{t}(x, y) d \mu(y) d \mu(x)\right. \\
& \left.\quad+2 \int_{M} f(x)^{2}\left(1-T_{t} 1(x)\right) d \mu(x)\right\} \\
\geq & \frac{1}{2 t} \int_{M} \int_{B(x, r)}(f(x)-f(y))^{2} p_{t}(x, y) d \mu(y) d \mu(x) .
\end{aligned}
$$


For any $r<\delta^{1+1 / \beta} r_{0}$, let $t=(r / \delta)^{\beta}$ so that $t<\delta r_{0}^{\beta}$. For such $r$ and $t$, since

$$
d(x, y)<r=\delta t^{1 / \beta}
$$

for almost all $x \in M$ and $y \in B(x, r)$, we can apply (NLE) in (3.4) to obtain that

$$
\begin{aligned}
\mathcal{E}(f) & \geq \frac{c_{0}}{2} t^{-(1+\alpha / \beta)} \int_{M} \int_{B(x, r)}(f(x)-f(y))^{2} d \mu(y) d \mu(x) \\
& =c r^{-(\alpha+\beta)} \int_{M} \int_{B(x, r)}(f(x)-f(y))^{2} d \mu(y) d \mu(x) .
\end{aligned}
$$

Let us verify that (3.5) also holds for $\delta^{1+1 / \beta} r_{0} \leq r<r_{0}$ (assuming $r_{0}<\infty$ ). Since $\mu$ satisfies the doubling condition, any ball of center $x_{0}$ and radius $r$ can be covered by a finite number (independent of $x_{0}$ and $r, r_{0}$ ) of balls of radius $\delta^{1+1 / \beta} r_{0}$. Applying (3.5) for each of these balls and adding up, we see that (3.5) holds for any $0<r<r_{0}$. Finally, taking supremum in $r$, we obtain from (3.5) that

$$
\mathcal{E}(f) \geq c W_{\beta / 2}(f) .
$$

Combining (3.5) and (2.8), we arrive at (3.2).

Theorem 3.3. Let $(M, d)$ be a metric space with a lower $\alpha$-regular measure $\mu$. Assume that the heat kernel $p_{t}(x, y)$ of the Dirichlet form $(\mathcal{E}, \mathcal{F})$ exists and satisfies $(N L E)$. Then the following upper estimate holds

$$
p_{t}(x, y) \leq \frac{c}{\min \left(t^{\alpha / \beta}, r_{0}^{\alpha}\right)}
$$

for all $t>0$ and $\mu$-almost all $x, y \in M$.

Proof. The conclusion immediately follows from Theorem 3.2 by a result in [7], which extends to the present setting the classical argument by Nash [27].

The estimate (DUE) is called a diagonal upper estimate because, in the setting when $p_{t}(x, y)$ is continuous in $x, y$, it is equivalent to the same estimate on the diagonal $x=y$ :

$$
p_{t}(x, x) \leq \frac{c}{\min \left(t^{\alpha / \beta}, r_{0}^{\alpha}\right)}
$$

by noting that, using the semigroup property and Cauchy-Schwarz inequality,

$$
\begin{aligned}
p_{t}(x, y) & =\int_{M} p_{t / 2}(x, z) p_{t / 2}(z, y) d \mu(z) \\
& \leq\left(\int_{M} p_{t / 2}(x, z)^{2} d \mu(z)\right)^{1 / 2}\left(\int_{M} p_{t / 2}(z, y)^{2} d \mu(z)\right)^{1 / 2} \\
& =\left(p_{t}(x, x) p_{t}(y, y)\right)^{1 / 2} .
\end{aligned}
$$

Remark 3.4. If $M$ is unbounded, we can take $r_{0}=\operatorname{diam}(M)=\infty$. Then (DUE) is reduced to

$$
p_{t}(x, y) \leq c t^{-\alpha / \beta}
$$

Corollary 3.5. Let $M$ be a Riemannian manifold, $d$ be the geodesic distance, $\mu$ be the Riemannian measure and $(\mathcal{E}, \mathcal{F})$ be the classical Dirichlet form on $M$, that is,

$$
\mathcal{E}(f)=\int_{M}|\nabla f|^{2} d \mu .
$$

Assume that $\mu$ is lower $\alpha$-regular and set $\beta=2$. Then $(N L E) \Longrightarrow(U E)$. 
Proof. Indeed, by Theorem 3.2, we have $(N L E) \Longrightarrow(D U E)$, that is,

$$
p_{t}(x, x) \leq c t^{-\alpha / 2},
$$

for all $t<r_{0}^{2}$ and $x \in M$. By [13], the on-diagonal estimate (3.9) on a manifold implies the off-diagonal upper bound:

$$
p_{t}(x, y) \leq \frac{c}{t^{\alpha / 2}} \exp \left(-\frac{d(x, y)^{2}}{c^{\prime} t}\right)
$$

for all $x, y \in M$ and $t<r_{0}^{2}$, for some $c, c^{\prime}>0$.

We make the following conjecture.

Conjecture 3.6. Let $(M, d, \mu)$ be a separable metric measure space with a lower $\alpha$-regular measure $\mu$ and $(\mathcal{E}, \mathcal{F})$ be a local regular Dirichlet form in $L^{2}(M, \mu)$. Then $(N L E) \Longrightarrow$ (UE).

In the next section, we will prove a version of this conjecture when a somewhat stronger version of (NLE) holds.

We finish this section with an example showing that the condition of the lower regularity of measure $\mu$ in Theorem 3.3 cannot be dropped.

Example 3.7. Let $M$ be a manifold obtained by gluing together $\mathbb{R}^{3}$ and $\mathbb{R}_{+} \times \mathbb{S}^{2}$, where $\mathbb{S}^{2}$ is the unit sphere in $\mathbb{R}^{3}$. More precisely, assume that $M$ is a complete 3-dimensional manifold such that $M$ is disjoint union of a compact set $K$, and open sets $E_{1}$ and $E_{2}$, where $E_{1}$ is isometric to $\mathbb{R}_{+} \times \mathbb{S}^{2}$, and $E_{2}$ isometric to $\mathbb{R}^{3} \backslash B_{0}$ where $B_{0}$ is a ball in $\mathbb{R}^{3}$, see Figure 1.

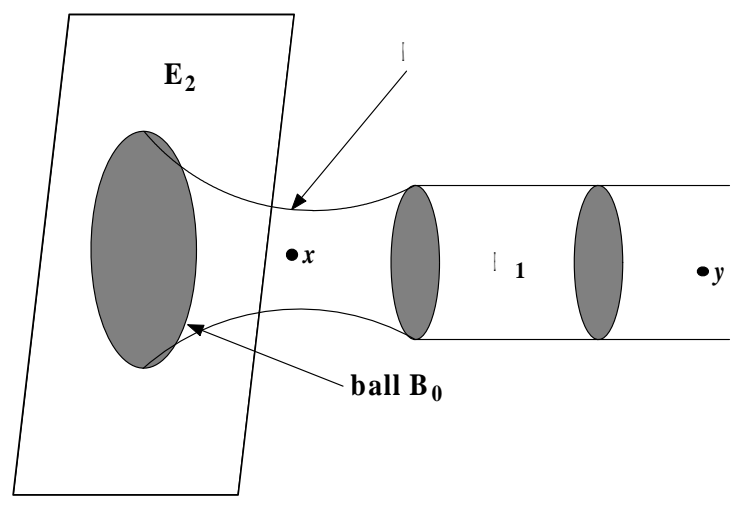

Figure 1. $M=E_{1} \cup E_{2} \cup K$

Let $\mu$ be the Riemannian measure on $M, d$ be the geodesic distance, and $\mathcal{E}$ be defined as in (3.8), which is local and regular. Set $r_{0}=\infty$. Obviously, $\mu$ is not lower $\alpha$-regular for any $\alpha$ (indeed, for small $r, \mu(B(x, r)) \asymp r^{3}$ whereas for large $r$ there are balls with $\mu(B(x, r)) \asymp r)$. It follows from [18, Example 3] that the heat kernel $p_{t}$ on $M$ satisfies the following lower estimate:

$$
p_{t}(x, y) \geq \frac{c}{t^{3 / 2}} \exp \left(-\frac{d(x, y)^{2}}{c^{\prime} t}\right),
$$

for all $t>0$ and $x, y \in M$, where $c, c^{\prime}>0$. Thus, (NLE) is true with $\alpha=3$ and $\beta=2$ (and no other choice of $\alpha$ and $\beta$ will do). On the other hand, if $x=y \in E_{1}$ with $d(x, K)=\sqrt{t}$ for large $t$, then it follows from [18, Example 3] that

$$
p_{t}(x, x) \geq \frac{c}{t^{1 / 2}} \gg \frac{c}{t^{3 / 2}} .
$$


Hence, the diagonal upper bound of $p_{t}$

$$
p_{t}(x, x) \leq \frac{c}{t^{3 / 2}}
$$

fails for such $x$ and $t$.

\section{LOCAL LOWER ESTIMATES IMPLY FULL UPPER BOUNDS}

We say that condition (UE) holds if

The heat kernel $p_{t}(x, y)$ exists and satisfies the upper estimate

$$
p_{t}(x, y) \leq c t^{-\alpha / \beta} \exp \left(-c^{\prime}\left(\frac{d(x, y)}{t^{1 / \beta}}\right)^{\frac{\beta}{\beta-1}}\right),
$$

for all $0<t<r_{0}^{\beta}$ and $\mu$-almost all $x, y \in M$, where $c, c^{\prime}>0$.

From now on we assume that the form $(\mathcal{E}, \mathcal{F})$ is local and regular. For any regular Dirichlet form $(\mathcal{E}, \mathcal{F})$, there exists an associated Hunt process $\left(\left\{X_{t}\right\}_{t>0},\left\{\mathbb{P}_{x}\right\}_{x \in M}\right)$, see $\left[12\right.$, Theorem 7.2.1]. If in addition $(\mathcal{E}, \mathcal{F})$ is local, then $X_{t}$ is a diffusion, that is, the path $t \rightarrow X_{t}$ is continuous almost surely [12, Theorem 7.2.2]. By the transition density of the process $X_{t}$, we mean a measurable function $\widetilde{p}_{t}(x, y)$ defined pointwise on $(0, \infty) \times M \times M$ such that

$$
\mathbb{E}_{x} f\left(X_{t}\right)=\int_{M} \widetilde{p}_{t}(x, y) f(y) d \mu(y)
$$

for all $x \in M, t>0$ and any bounded Borel function $f$. For any such function $f$, set

$$
P_{t} f(x):=\mathbb{E}_{x} f\left(X_{t}\right) \quad(x \in M, t>0) .
$$

Then $\left\{P_{t}\right\}_{t \geq 0}$ is a semigroup on bounded Borel functions. It is well-known [12] that

$$
T_{t} f(x)=P_{t} f(x) \quad \text { a.e. }
$$

for all $t>0$ and all bounded Borel functions $f$. This implies that if the heat kernel $p_{t}(x, y)$ and the transition density $\widetilde{p}_{t}(x, y)$ exist, then

$$
p_{t}(x, y)=\widetilde{p}_{t}(x, y)
$$

for all $t>0$ and $\mu$-almost all $x, y \in M$. Let us emphasize that unlike the heat kernel, the transition density is defined for all $x, y \in M$.

We say that $N \subset M$ is the negligible set, if $\mu(N)=0$ and

$$
\mathbb{P}_{x}\left(X_{t} \in N \text { or } X_{t-} \in N \text { for some } t \geq 0\right)=0 \quad \text { for all } x \in M \backslash N \text {. }
$$

It follows from [2, Proposition 4.14, Corollary 4.15] or [16] that if the heat kernel exists and satisfies (DUE), then the transition density $\widetilde{p}_{t}(x, y)$ satisfies (DUE) for all $x, y \in M \backslash N$ and $t>0$, where $N$ is a negligible set.

Let $\Omega$ be an open subset of $M$, and define

$$
\mathcal{F}_{\Omega}=\left\{f \in \mathcal{F}:\left.f\right|_{M \backslash \Omega}=0\right\} .
$$

If $(\mathcal{E}, \mathcal{F})$ is a regular Dirichlet form on $L^{2}(M, \mu)$, then the form $\left(\mathcal{E}, \mathcal{F}_{\Omega}\right)$ is also a regular Dirichlet form on $L^{2}(\Omega, \mu)\left[12\right.$, Theorem 4.4.3, p.154]. Let $\left(\left\{X_{t}\right\}_{t \geq 0},\left\{\mathbb{P}_{x}^{\Omega}\right\}_{x \in M}\right)$ be a (killed) Hunt process associated with $\left(\mathcal{E}, \mathcal{F}_{\Omega}\right)$. Then, for any bounded Borel function $f$, and for all $x \in M, t>0$,

$$
P_{t}^{\Omega} f(x):=\mathbb{E}_{x}^{\Omega}\left(f\left(X_{t}\right)\right)=\mathbb{E}_{x}\left(\mathbf{1}_{\left\{t<\tau_{\Omega}\right\}} f\left(X_{t}\right)\right)
$$

where

$$
\tau_{\Omega}=\inf \left\{t>0: X_{t} \notin \Omega\right\}
$$


the first exit time from $\Omega$, see [12, (4.1.2), p.135]. Assume that the transition density for the killed process on $\Omega$ exists for any open subset $\Omega$, and denote it by $\widetilde{p}_{t}^{\Omega}(x, y)$. Clearly $\widetilde{p}_{t}^{\Omega}(x, y)=0$ for all $t>0$ if $x \notin \Omega$ or $y \notin \Omega$. It follows from (4.3) that, for all $x \in M$ and all $t>0$,

$$
\widetilde{p}_{t}^{\Omega}(x, y) \leq \widetilde{p}_{t}(x, y)
$$

for $\mu$-almost all $y \in M$.

Taking $f=1$ in (4.3) and integrating in $e^{-\lambda t} d t$ over $(0, \infty)$, we obtain that

$$
\begin{aligned}
\lambda \int_{0}^{\infty} e^{-\lambda t} P_{t}^{\Omega} 1(x) d t & =\lambda \int_{0}^{\infty} e^{-\lambda t} \mathbb{E}_{x}\left(\mathbf{1}_{\left\{t<\tau_{\Omega}\right\}}\right) d t \\
& =\mathbb{E}_{x}\left(\lambda \int_{0}^{\tau_{\Omega}} e^{-\lambda t} d t\right) \\
& =1-\mathbb{E}_{x}\left(e^{-\lambda \tau_{\Omega}}\right) .
\end{aligned}
$$

Therefore,

$$
\mathbb{E}_{x}\left(e^{-\lambda \tau_{\Omega}}\right)=1-\lambda \int_{0}^{\infty} e^{-\lambda t} P_{t}^{\Omega} 1(x) d t
$$

for all $x \in M$ and $\lambda \geq 0$, and for any open subset $\Omega$ of $M$. In the remainder of this section, we always set

$$
r_{0}:=\operatorname{diam}(M) .
$$

In order to obtain off-diagonal upper estimates of the heat kernel $p_{t}(x, y)$, we assume the following local lower estimate of the heat kernel:

(LLE): For any ball $B$, the local heat kernel $p_{t}^{B}(x, y)$ exists. Moreover, there exist some $c_{0}>0, \beta>1, \delta \in(0,1)$ such that, for all $x_{0} \in M, 0<r<r_{0}$ and all $t \leq \delta r^{\beta}$,

$$
p_{t}^{B\left(x_{0}, r\right)}(x, y) \geq c_{0} t^{-\alpha / \beta},
$$

for $\mu$-almost all $x, y \in B\left(x_{0}, \delta t^{1 / \beta}\right)$, where $\alpha$ is the same as in (2.4).

Roughly speaking, the condition (LLE) says that the Dirichlet heat kernel $p_{t}^{B\left(x_{0}, r\right)}(x, y)$ satisfies the near-diagonal lower bound for $x, y$ close to the center of the ball, see Figure 2 .

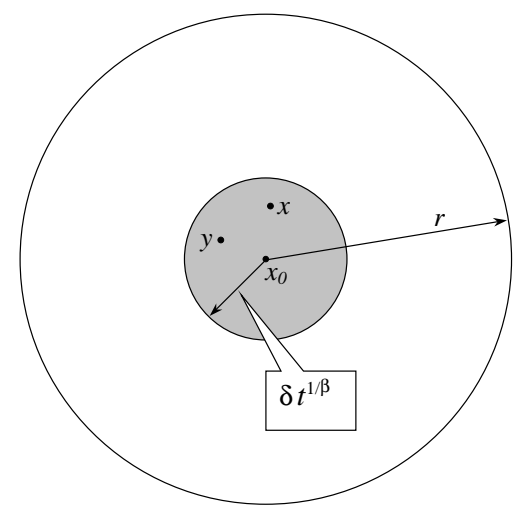

Figure 2. Balls $B\left(x_{0}, r\right)$ and $B\left(x_{0}, \delta t^{1 / \beta}\right)$

If $p_{t}^{B\left(x_{0}, r\right)}(x, y)$ is continuous in $x, y$ for any ball $B\left(x_{0}, r\right)$, then we can rephrase the local lower estimate in a simpler way: there exist some $c_{0}>0, \beta>1, \delta \in(0,1)$ such that, for 
all $x \in M, 0<r<r_{0}$ and all $t \leq \delta r^{\beta}$,

$$
p_{t}^{B(x, r)}(x, y) \geq c_{0} t^{-\alpha / \beta},
$$

for all $y \in B\left(x, \delta t^{1 / \beta}\right)$.

Lemma 4.1. Let $(\mathcal{E}, \mathcal{F})$ be a Dirichlet form on a separable metric space $M$. If (LLE) holds, then the (global) heat kernel $p_{t}(x, y)$ of $(\mathcal{E}, \mathcal{F})$ exists and satisfies $(N L E)$ with $r_{0}=$ $\operatorname{diam}(M)$.

Proof. Observe that the heat kernel $p_{t}$ of $(\mathcal{E}, \mathcal{F})$ exists under the hypothesis (LLE). Indeed, if $r_{0}<\infty$ then $M$ is a metric ball $B$ and, hence, $p_{t}=p_{t}^{B}$. If $r_{0}=\infty$ then, for a fixed $x_{0} \in M$, the sequence $\left\{p_{t}^{B\left(x_{0}, n\right)}\right\}_{n=1}^{\infty}$ is increasing in $n$, its limit is finite almost everywhere and is the heat kernel $p_{t}$ of $(\mathcal{E}, \mathcal{F})$, see [16, Lemma 4.1].

It remains to show $p_{t}(x, y)$ satisfies (NLE). Fix $t<\delta r_{0}^{\beta}$, and let $r$ be such that $t=\delta r^{\beta}$. By (LLE), we have that, for any $z \in M$, there is a set $N_{z} \subset M$ such that $\mu\left(N_{z}\right)=0$ and

$$
p_{t}^{B(z, r)}(x, y) \geq c_{0} t^{-\alpha / \beta}
$$

for all $x, y \in B\left(z, \delta t^{1 / \beta}\right) \backslash N_{z}$. By adjusting $N_{z}$, we can assume that

$$
p_{t}(x, y) \geq p_{t}^{B(z, r)}(x, y)
$$

also for all $x, y \in B\left(z, \delta t^{1 / \beta}\right) \backslash N_{z}$. Hence, for all $x, y \in B\left(z, \delta t^{1 / \beta}\right) \backslash N_{z}$,

$$
p_{t}(x, y) \geq c_{0} t^{-\alpha / \beta} \text {. }
$$

Consider the subsets of $M \times M$

$$
A=\left\{(x, y) \in M \times M: d(x, y)<\delta t^{1 / \beta}\right\}
$$

and

Clearly we have

$$
A_{z}=\left\{(x, y) \in M \times M: x, y \in B\left(z, \delta t^{1 / \beta}\right)\right\} .
$$

$$
A \subset \bigcup_{z \in M} A_{z}
$$

because, for any $(x, y) \in A$, we see that $x, y \in B\left(x, \delta t^{1 / \beta}\right)$ and hence, $(x, y) \in A_{x}$. Now, since each set $A_{z}$ is open in $M \times M$ and $M \times M$ has a countable base, there is a countable family $\left\{A_{z_{k}}\right\}_{k=1}^{\infty}$ such that

$$
A \subset \bigcup_{k=1}^{\infty} A_{z_{k}} .
$$

Since (4.8) holds for all $(x, y) \in A_{z_{k}} \backslash N_{z_{k}}$ for any $k$, we obtain that the same is true for any $(x, y) \in \cup_{k \geq 1} A_{z_{k}} \backslash N$, where

$$
N:=\bigcup_{k=1}^{\infty} N_{z_{k}}
$$

has zero measure, and that (4.8) holds for all $(x, y) \in A \backslash N$. Therefore (NLE) follows.

The next theorem is our main result in this paper.

Theorem 4.2. Let $(M, d, \mu)$ be a separable metric measure space and measure $\mu$ be lower $\alpha$-regular. Let $(\mathcal{E}, \mathcal{F})$ be a local regular Dirichlet form in $L^{2}(M, \mu)$. Then the following equivalence holds:

$$
(L L E) \Longleftrightarrow(U E)+(N L E)
$$


Proof. We first prove the implication " $(L L E) \Rightarrow(U E)+(N L E)$ ". By Lemma 4.1, (LLE) implies that the heat kernel $p_{t}(x, y)$ of $(\mathcal{E}, \mathcal{F})$ exists and satisfies (NLE). Thus we only need to prove $(U E)$ assuming that (LLE) holds. Observe that (LLE) implies that there exists a negligible set $N$ such that, for all $x_{0} \in M, 0<r<r_{0}$ and all $t \leq \delta r^{\beta}$,

$$
\widetilde{p}_{t}^{B\left(x_{0}, r\right)}(x, y) \geq c_{0} t^{-\alpha / \beta}
$$

for all $x, y \in B\left(x_{0}, \delta t^{1 / \beta}\right) \backslash N$, see [16]. Let us show that, for all $x \in M \backslash N, 0<r<r_{0}$ and all $t>0$,

$$
\mathbb{P}_{x}\left(\tau_{B(x, r)} \leq t\right) \leq c \exp \left(-c^{\prime}\left(\frac{r}{t^{1 / \beta}}\right)^{\frac{\beta}{\beta-1}}\right)
$$

The proof of (4.10) given here is motivated by [16, Theorem 9.1] or [2]. We first prove that there exists some $\varepsilon \in(0,1)$ such that, for all $x \in M \backslash N, 0<r<r_{0}$ and $\lambda \geq r^{-\beta}$,

$$
\mathbb{E}_{x}\left(e^{-\lambda \tau_{B(x, r)}}\right) \leq \varepsilon
$$

To see this, fix $x \in M \backslash N$ and $0<r<r_{0}$, and set $\tau=\tau_{B(x, r)}$. For $0<t<\delta r^{\beta}$, we see from (4.9) with $x_{0}=x$ that

$$
\begin{aligned}
P_{t}^{B(x, r)} 1(x) & =\int_{M} \widetilde{p}_{t}^{B(x, r)}(x, y) d \mu(y) \\
& \geq \int_{B\left(x, \delta t^{1 / \beta}\right) \backslash N} \widetilde{p}_{t}^{B(x, r)}(x, y) d \mu(y) \\
& \geq c_{0} t^{-\alpha / \beta} \mu\left(B\left(x, \delta t^{1 / \beta}\right)\right) \geq c>0 .
\end{aligned}
$$

It follows that, for $\lambda \geq r^{-\beta}$,

$$
\begin{aligned}
\lambda \int_{0}^{\infty} e^{-\lambda t} P_{t}^{B(x, r)} 1(x) d t & \geq \lambda \int_{0}^{\delta r^{\beta}} e^{-\lambda t} P_{t}^{B(x, r)} 1(x) d t \\
& \geq c \lambda \int_{0}^{\delta r^{\beta}} e^{-\lambda t} d t=c\left(1-e^{-\delta \lambda r^{\beta}}\right) \geq c^{\prime}>0 .
\end{aligned}
$$

Therefore, by (4.5),

$$
\mathbb{E}_{x}\left(e^{-\lambda \tau}\right)=1-\lambda \int_{0}^{\infty} e^{-\lambda t} P_{t}^{B(x, r)} 1(x) d t \leq 1-c^{\prime},
$$

proving (4.11).

Now we show that (4.11) implies (4.10). For $r_{0}=\infty$, this was proved in $[2,16]$. For $r_{0}<\infty$, the proof is the same. To see this, we show that, for $x \in M \backslash N$ and $0<r<r_{0}$,

$$
\mathbb{E}_{x}\left(e^{-\lambda \tau}\right) \leq c \exp \left(-c^{\prime} \lambda^{1 / \beta} r\right)
$$

for $\lambda>0$, where $c, c^{\prime}>0$ are independent of $x, r$ and $\lambda$. Indeed, let $\rho=r / n$ where $n \geq 1$ will be chosen below. Set $\tau_{k}=\tau(x, k \rho)$, the first exit time from the ball $B_{k}:=B(x, k \rho)$, for $k=1, \cdots, n$. By the strong Markov property, we have that

$$
\mathbb{E}_{x}\left(e^{-\lambda \tau_{k+1}}\right)=\mathbb{E}_{x}\left(e^{-\lambda \tau_{k}} e^{-\lambda\left(\tau_{k+1}-\tau_{k}\right)}\right)=\mathbb{E}_{x}\left(e^{-\lambda \tau_{k}} \mathbb{E}_{X_{\tau_{k}}} e^{-\lambda \tau_{k+1}}\right) .
$$


Note that $X_{\tau_{k}} \in \partial B_{k} \backslash N$ with $\mathbb{P}_{x}$-probability 1 , and $\tau_{k+1} \geq \tau_{B(y, \rho)}$ for any $y \in \partial B_{k}$. We have from (4.13) and (4.11) that, for $\lambda \geq \rho^{-\beta}$,

$$
\begin{aligned}
\mathbb{E}_{x}\left(e^{-\lambda \tau_{k+1}}\right) & \leq \mathbb{E}_{x}\left(e^{-\lambda \tau_{k}}\right) \sup _{y \in \partial B_{k} \backslash N} \mathbb{E}_{y}\left(e^{-\lambda \tau_{B(y, \rho)}}\right) \\
& \leq \varepsilon \mathbb{E}_{x}\left(e^{-\lambda \tau_{k}}\right) \quad(1 \leq k \leq n-1) .
\end{aligned}
$$

Now choose the largest integer $n$ such that $\lambda \geq \rho^{-\beta}=(n / r)^{\beta}$, that is,

$$
n^{\beta} \leq \lambda r^{\beta} .
$$

(We assume that $\lambda r^{\beta}$ is large enough; otherwise (4.12) automatically holds.) Therefore,

$$
\mathbb{E}_{x}\left(e^{-\lambda \tau}\right) \leq \varepsilon^{n}=e^{-n \log \frac{1}{\varepsilon}} \leq e^{-\left(\lambda^{1 / \beta} r-1\right) \log \frac{1}{\varepsilon}},
$$

proving (4.12). By (4.12), we have that

$$
\begin{aligned}
\mathbb{P}_{x}(\tau \leq t) & =\mathbb{P}_{x}\left(e^{-\lambda \tau} \geq e^{-\lambda t}\right) \leq e^{\lambda t} \mathbb{E}_{x}\left(e^{-\lambda \tau}\right) \\
& \leq c \exp \left(\lambda t-c^{\prime} \lambda^{1 / \beta} r\right) \\
& \leq c \exp \left(-c^{\prime \prime}\left(r^{\beta} t^{-1}\right)^{1 /(\beta-1)}\right)
\end{aligned}
$$

by taking $\lambda$ such that $\lambda t=\frac{1}{2} c^{\prime} \lambda^{1 / \beta} r$. Thus (4.10) holds.

Finally, fix $x_{0}, y_{0} \in M\left(x_{0} \neq y_{0}\right)$ and let $r=\frac{1}{2} d\left(x_{0}, y_{0}\right)$. Then, for almost all $x \in B\left(x_{0}, r\right)$ and $y \in B\left(y_{0}, r\right)$,

$$
\begin{aligned}
p_{t}(x, y) & \leq \mathbb{P}_{x}\left(\tau_{B\left(x_{0}, r\right)} \leq \frac{t}{2}\right) \sup _{t / 2 \leq s \leq t} \operatorname{esssup}_{u \in B\left(x_{0}, 2 r\right)} p_{s}(u, y) \\
& +\mathbb{P}_{y}\left(\tau_{B\left(y_{0}, r\right)} \leq \frac{t}{2}\right) \sup _{t / 2 \leq s \leq t} \operatorname{esssup}_{v \in B\left(y_{0}, 2 r\right)} p_{s}(v, x),
\end{aligned}
$$

see [16]. By (4.10), we see that, for any $x \in B\left(x_{0}, r / 2\right) \backslash N$,

$$
\mathbb{P}_{x}\left(\tau_{B\left(x_{0}, r\right)} \leq \frac{t}{2}\right) \leq \mathbb{P}_{x}\left(\tau_{B(x, r / 2)} \leq \frac{t}{2}\right) \leq c \exp \left(-c^{\prime}\left(\frac{r}{t^{1 / \beta}}\right)^{\beta /(\beta-1)}\right)
$$

Similarly, for any $y \in B\left(y_{0}, r / 2\right) \backslash N$,

$$
\mathbb{P}_{y}\left(\tau_{B\left(y_{0}, r\right)} \leq \frac{t}{2}\right) \leq c \exp \left(-c^{\prime}\left(\frac{r}{t^{1 / \beta}}\right)^{\beta /(\beta-1)}\right) .
$$

Noting that, for $\mu$-almost all $u, y \in M$ and $t / 2 \leq s \leq t$, we have from (DUE) that

$$
p_{s}(u, y) \leq c t^{-\alpha / \beta} \quad \text { if } t<r_{0}^{\beta} .
$$

Therefore, we combine (4.16), (4.17) and (4.18) to obtain (UE).

For the opposite implication, we prove a stronger claim. For this, we introduce a condition $(\Phi \mathrm{UE})$ as follows:

( $\Phi \mathbf{U E}): \quad$ There exists a bounded positive function $\Phi$ on $[0, \infty)$ satisfying

$$
\sup _{s \geq 0} s^{\alpha} \Phi(s)<\infty
$$

such that the heat kernel $p_{t}(x, y)$ of $(\mathcal{E}, \mathcal{F})$ satisfies the estimate

$$
p_{t}(x, y) \leq t^{-\alpha / \beta} \Phi\left(\frac{d(x, y)}{t^{1 / \beta}}\right)
$$

for all $t<r_{0}^{\beta}$ and $\mu$-almost all $x, y \in M$. 
Obviously, (UE) $\Longrightarrow(\Phi \mathrm{UE})$ because one can take $\Phi(s)=c \exp \left(-c^{\prime} s^{\frac{\beta}{\beta-1}}\right)$. We claim that if $(\mathcal{E}, \mathcal{F})$ is a regular Dirichlet form (not necessarily local) then

$$
(\mathrm{NLE})+(\Phi \mathrm{UE}) \Longrightarrow(\mathrm{LLE}) \text {. }
$$

Note that $(\Phi U E) \Longrightarrow(D U E)$ and (DUE) implies the Nash inequality (3.2) for all $f \in \mathcal{F}$. In particular, this inequality holds also for all $f \in \mathcal{F}_{\Omega}$, which implies that $p_{t}^{\Omega}(x, y)$ exists by the results of [7] and [16].

Next, let us apply the following inequality

$$
p_{t}(x, y) \leq p_{t}^{\Omega}(x, y)+\sup _{0<s \leq t} \operatorname{esssup}_{z \in \Omega^{c}} p_{s}(y, z)
$$

which is true for all open $\Omega \subset M$, for all $t>0$ and $\mu$-almost all $x, y \in \Omega$, see [16, Lemma 8.1]. Fix some $0<\delta^{\prime} \leq \delta / 2$ to be specified below where $\delta$ is the constant from (NLE). Also, fix some $x_{0} \in M, r \in\left(0, r_{0}\right), s>0$, and $0<t \leq \delta^{\prime} r^{\beta}$. For all $z \in B\left(x_{0}, r\right)^{c}$ and $y \in B\left(x_{0}, \delta^{\prime} t^{1 / \beta}\right)$, we have

$$
d(y, z) \geq d\left(z, x_{0}\right)-d\left(x_{0}, y\right) \geq r-\delta^{\prime} t^{1 / \beta} \geq\left(1-\left(\delta^{\prime}\right)^{1+1 / \beta}\right) r \geq \text { const } r .
$$

Then by ( $\Phi \mathrm{UE})$, we have, for $\mu$-almost all $z \in B\left(x_{0}, r\right)^{c}$ and $y \in B\left(x_{0}, \delta^{\prime} t^{1 / \beta}\right)$,

$$
\begin{aligned}
p_{s}(y, z) & \leq s^{-\alpha / \beta} \Phi\left(\frac{d(y, z)}{s^{1 / \beta}}\right) \\
& =d(y, z)^{-\alpha}\left(\frac{d(y, z)}{s^{1 / \beta}}\right)^{\alpha} \Phi\left(\frac{d(y, z)}{s^{1 / \beta}}\right) \\
& \leq d(y, z)^{-\alpha} \sup _{\xi \geq 0} \xi^{\alpha} \Phi(\xi) \leq c r^{-\alpha} \leq c\left(\delta^{\prime}\right)^{\alpha / \beta} t^{-\alpha / \beta} .
\end{aligned}
$$

Choosing $\delta^{\prime}$ to be small enough, we obtain that for $\mu$-almost all $y \in B\left(x_{0}, \delta^{\prime} t^{1 / \beta}\right)$,

$$
\underset{z \in B\left(x_{0}, r\right)^{c}}{\operatorname{esssup}} p_{s}(y, z) \leq \frac{c_{0}}{2} t^{-\alpha / \beta},
$$

where $c_{0}$ is the constant from (NLE). Applying (4.20) with $\Omega=B\left(x_{0}, r\right)$ and using (NLE), we obtain that for $\mu$-almost all $x, y \in B\left(x_{0}, \delta^{\prime} t^{1 / \beta}\right)$

$$
c_{0} t^{-\alpha / \beta} \leq p_{t}(x, y) \leq p_{t}^{B\left(x_{0}, r\right)}(x, y)+\frac{c_{0}}{2} t^{-\alpha / \beta},
$$

which implies that

$$
p_{t}^{B\left(x_{0}, r\right)}(x, y) \geq \frac{c_{0}}{2} t^{-\alpha / \beta} .
$$

Hence, we have proved (LLE) with the parameter $\delta^{\prime}$.

Remark 4.3. Assume that the hypothesis in Theorem 4.2 hold. If in addition $(M, d)$ satisfies the chain condition, then we obtain from Proposition 3.1 the following result: the local lower estimate (LLE) is equivalent to the two-sided estimate

$$
p_{t}(x, y) \asymp t^{-\alpha / \beta} \exp \left(-c^{\prime}\left(\frac{d(x, y)}{t^{1 / \beta}}\right)^{\frac{\beta}{\beta-1}}\right),
$$

for all $0<t<r_{0}^{\beta}$ and $\mu$-almost all $x, y \in M$. Here the value of the constant $c^{\prime}$ may be different for the upper and lower estimates. 
Remark 4.4. In Theorem 4.2, we do not assume that the diffusion $\left(\left\{X_{t}\right\}_{t \geq 0},\left\{\mathbb{P}_{x}\right\}_{x \in M}\right)$ is stochastically complete, that is

$$
\mathbb{P}_{x}\left(X_{t} \in M\right)=1 \quad \text { for all } x \in M, t>0 .
$$

This condition is usually assumed in most literature on the heat kernel estimates.

Remark 4.5. Theorem 4.2 provides a new way of obtaining two-sided estimates of the heat kernel $p_{t}(x, y)$ from the parabolic Harnack inequality. Indeed, by the standard argument (see $[1,28,31])$, the parabolic Harnack inequality implies (LLE), whence (UE) and (NLE) follows.

For the next statement, we need the following condition, which is referred to as the time independent upper estimate:

$$
p_{t}(x, y) \leq c d(x, y)^{-\alpha} \text { for all } t<r_{0}^{\beta} \text { and } \mu \text {-a.a. } x, y \in M .
$$

It is easy to see that $(\Phi \mathrm{UE}) \Longrightarrow(\mathrm{TIUE})$.

Theorem 4.6. Let $(M, d, \mu)$ be a separable metric measure space and let $(\mathcal{E}, \mathcal{F})$ be a local regular Dirichlet form in $L^{2}(M, \mu)$. If measure $\mu$ is lower $\alpha$-regular, then

$$
(T I U E)+(N L E) \Longleftrightarrow(U E)+(N L E) .
$$

Proof. The direction $\Leftarrow$ is obvious because (TIUE) coincides with ( $\Phi \mathrm{UE})$ with function $\Phi(s)=c s^{-\alpha}$ that satisfies (4.19) (despite this function is unbounded).

To prove the opposite implication, observe that, by Corollary 3.3, (NLE) implies (DUE), which together with (TIUE) yields

$$
p_{t}(x, y) \leq c \min \left\{t^{-\alpha / \beta}, d(x, y)^{-\alpha}\right\}
$$

for all $t<r_{0}^{\beta}$ and $\mu$-a.a. $x, y \in M$. Obviously, (4.21) is equivalent to ( $\left.\Phi \mathrm{UE}\right)$ with the function

$$
\Phi(s)=c \begin{cases}s^{-\alpha}, & s>1 \\ 1, & s \leq 1 .\end{cases}
$$

which is clearly bounded and satisfies (4.19). By the second part of the proof of Theorem 4.2, ( $\Phi \mathrm{UE})+(\mathrm{NLE})$ imply (LLE). Then, by Theorem 4.2, (LLE) implies (UE).

The next example shows that if $(\mathcal{E}, \mathcal{F}$ ) is not local (whilst the other conditions in Theorem 4.2 are still true), then (LLE) does not imply (UE).

Example 4.7. Let $(M, d, \mu)$ be a metric measure space with a Dirichlet form $(\mathcal{E}, \mathcal{F})$ whose heat kernel $p_{t}(x, y)$ is stochastically complete and satisfies the estimates

$$
t^{-\alpha / \beta} \Phi_{1}\left(t^{-1 / \beta} d(x, y)\right) \leq p_{t}(x, y) \leq t^{-\alpha / \beta} \Phi_{2}\left(t^{-1 / \beta} d(x, y)\right)
$$

for all $0<t<r_{0}^{\beta}$ and $\mu$-almost all $x, y \in M$, where $r_{0}=\operatorname{diam}(M)$, and $\alpha>0, \beta>1$ and $\Phi_{1}$ and $\Phi_{2}$ are positive decreasing functions on $[0, \infty)$. For example, for some basic fractals such as the Sierpinski gaskets or the Sierpinski carpets, it is known that (4.23) holds with

$$
\Phi_{i}(s)=c_{i} \exp \left(-c_{i}^{\prime} s^{\gamma_{i}}\right) \quad(i=1,2)
$$

for some $c_{i}, c_{i}^{\prime}, \gamma_{i}>0$, see for example [6], [4], or [21]. Under certain mild conditions on $\Phi_{1}$ and $\Phi_{2},(4.23)$ implies that the measure $\mu$ is $\alpha$-regular, see [17]. 
Let $H$ be the associated infinitesimal generator of the heat kernel $p_{t}$, defined by $(2.3)$ and (2.1). Then, for any $0<\sigma<1$, the heat kernel $p_{t}^{(\sigma)}$ corresponding to the fractional power $H^{\sigma}$ satisfies the estimate

$$
p_{t}^{(\sigma)} \asymp t^{-\alpha / \beta^{\prime}}\left(1+\frac{d(x, y)}{t^{1 / \beta^{\prime}}}\right)^{-\left(\alpha+\beta^{\prime}\right)},
$$

where $\beta^{\prime}=\sigma \beta$, for all $0<t<r_{0}^{\beta^{\prime}}$ and $\mu$-almost all $x, y \in M$. (see for example [15, 24]). The Dirichlet form $\left(\mathcal{E}^{(\sigma)}, \mathcal{F}\right)$ corresponding to $p_{t}^{(\sigma)}(x, y)$ is given by

$$
\mathcal{E}^{(\sigma)}(u)=\left(H^{\sigma} u, u\right)=\frac{1}{2} \int_{M} \int_{M}(u(x)-u(y))^{2} k(x, y) d \mu(y) d \mu(x)
$$

where

$$
k(x, y)=\frac{\sigma}{\Gamma(1-\sigma)} \int_{0}^{\infty} p_{t}(x, y) \frac{d t}{t^{\sigma+1}} \asymp d(x, y)^{-\left(\alpha+\beta^{\prime}\right)} .
$$

One can see that $\mathcal{E}^{(\sigma)}$ is regular but not local. By $(4.24)$, we see that $p_{t}^{(\sigma)}(x, y)$ does not satisfy (UE). However, it satisfies ( $\Phi U E)$ and (NLE), which implies (LLE) by the second part of the proof of Theorem 4.2. Therefore, (LLE) does not imply (UE) if $(\mathcal{E}, \mathcal{F})$ is not local.

To be more specific, let us consider $M=\mathbb{R}^{n}$ with the Lebesgue measure $\mu$ and the classical Dirichlet form $(\mathcal{E}, \mathcal{F})$ given by $(3.8)$. Then the Dirichlet form $\mathcal{E}^{(1 / 2)}$ is given by

$$
\mathcal{E}^{(1 / 2)}(f)=\frac{C_{n}}{2} \int_{\mathbb{R}^{n}} \int_{\mathbb{R}^{n}} \frac{(f(x)-f(y))^{2}}{|x-y|^{n+1}} d y d x
$$

where $C_{n}=\Gamma\left(\frac{n+1}{2}\right) / \pi^{(n+1) / 2}$, and its heat kernel admits the explicit formula

$$
p_{t}^{(1 / 2)}(x, y)=\frac{C_{n}}{t^{n}}\left(1+\frac{|x-y|^{2}}{t^{2}}\right)^{-\frac{n+1}{2}},
$$

that is, $p_{t}^{(1 / 2)}$ is the Cauchy-Poisson kernel. In this case, we have $\alpha=n$ and $\beta^{\prime}=1$. Note that the form $\mathcal{E}^{(1 / 2)}$ is regular but not local, and $p_{t}^{(1 / 2)}(x, y)$ does not satisfy (UE) although it satisfies (LLE).

\section{REFERENCES}

[1] D.G. Aronson, Bounds for the fundamental solution of a parabolic equation, Bull. Amer. Math. Soc. 73 (1967), 890-896.

[2] M.T. Barlow, Diffusions on fractals, Lectures on Probability Theory and Statistics, Lect. Notes Math., Springer, 1690 (1998), 1-121.

[3] M.T. Barlow, Heat kernels and sets with fractal structure, in Heat kernels and analysis on manifolds, graphs, and metric spaces, Contemporary Math. 338 (2003), 11-40.

[4] M.T. Barlow and R.F. Bass, Brownian motion and harmonic analysis on Sierpínski carpets, Canad. J. Math. (4) 51 (1999), 673-744.

[5] M. Barlow, T. Coulhon and T. Kumagai, Characterization of sub-Gaussian heat kernel estimates on strongly recurrent graphs, Comm. Pure Appl. Math. 58 (2005), 1642-1677.

[6] M.T. Barlow and E.A. Perkins, Brownian motion on the Sierpinski gasket, Probab. Theory Related Fields 79 (1988), 543-623.

[7] E.A. Carlen and S. Kusuoka and D.W. Stroock, Upper bounds for symmetric Markov transition functions, Ann. Inst. Heri. Poincarè-Probab. Statist. 23 (1987), 245-287.

[8] I. Chavel, Eigenvalues in Riemannian geometry, Academic Press, New York, 1984.

[9] T. Coulhon and A. Grigor'yan, Pointwise estimates for transition probabilities of random walks on infinite graphs, in Trends in Mathematics: Fractals in Graz 2001, ed. P.Grabner and W.Woess, Birkhäuser 2002, 119-134.

[10] E.B. Davies, Heat kernels and spectral theory, Cambridge University Press, 1989. 
[11] E.B. Fabes and D.W. Stroock, A new proof of Moser's parabolic Harnack inequality using the old ideas of Nash, Arch. Rational Mech. Anal. 96 (1986), 327-338.

[12] M. Fukushima, Y. Oshima and M. Takeda, Dirichlet forms and symmetric Markov processes, de Gruyter, Berlin, 1994.

[13] A. Grigor'yan, Gaussian upper bounds for the heat kernel on arbitrary manifolds, J. Diff. Geom. 45 (1997), 33-52.

[14] A. Grigor'yan, Estimates of heat kernels on Riemannian manifolds, London Math. Soc. Lecture Note Ser., 273 (1999), 140-225.

[15] A. Grigor'yan, Heat kernels and function theory on metric measure spaces, Contemporary Math. 338 (2003), 143-172.

[16] A. Grigor'yan, Heat kernels and exit time, preprint.

[17] A. Grigor'yan, J. Hu and K.-S. Lau, Heat kernels on metric-measure spaces and an application to semilinear elliptic equations, Trans. Amer. Math. Soc. 355 (2003), 2065-2095.

[18] A. Grigor'yan and L. Saloff-Coste, Heat kernel on connected sums of Riemannian manifolds, Math. Res. Lett. 6 (1999), 307-321.

[19] A. Grigor'yan and A. Telcs, Sub-Gaussian estimates of heat kernels on infinite graphs, Duke Math. J. 109 (2001), 451-510.

[20] A. Grigor'yan and A. Telcs, Harnack inequalities and sub-Gaussian estimates for random walks, Math. Ann. 324 (2002), 521-556.

[21] B.M. Hambly and T. Kumagai, Transition density estimates for diffusion processes on post critically finite self-similar fractals, Proc. London Math. Soc. (3) 79 (1999), 431-458.

[22] W. Hebisch and L. Saloff-Coste, On the relation between elliptic and parabolic Harnack inequalities, Ann. Inst. Fourier (Grenoble) 51 (2001), 1437-1481.

[23] J. Hu and T. Kumagai, Nash-type inequalities and heat kernels for non-local Dirichlet forms, Kyushu J. Math. (to appear).

[24] J Hu and M. Zähle, Jump processes and nonlinear fractional heat equations on fractals, Math. Nachr. 279 (2006), 150-163.

[25] J. Kigami, Analysis on Fractals, Cambridge University Press, 2001.

[26] J. Moser, A Harnack inequality for parabolic differential equations, Comm. Pure Appl. Math., 17 (1964), 101-134.

[27] J. Nash, Continuity of solutions of parabolic and elliptic equations, Amer. J. Math. 80 (1958), 931-954.

[28] F.O. Porper and S.D. Eidel'man, Two-side estimates of fundamental solutions of second-order parabolic equations and some applications, Russian Math. Surveys, 39 (1984), 119-178.

[29] L. Saloff-Coste, Aspects of Sobolev inequalities, LMS Lecture Notes Series 289 Cambridge Univ. Press, 2002.

[30] R. Schoen and S.-T Yau, Lectures on Differential Geometry, International Press, 1994.

[31] D.W. Stroock, Estimates on the heat kernel for the second order divergence form operators, in: Probability theory. Proceedings of the 1989 Singapore Probability Conference held at the National University of Singapore, June 8-16 1989, ed. L.H.Y. Chen, K.P. Choi, K. Hu and J.H. Lou, Walter De Gruyter, 1992, 29-44.

FAKUltät FÜR Mathematik, Universität Bielefeld, 33507 Bielefeld, Germany.

E-mail address: grigor@math.uni-bielefeld.de

Department of Mathematical Sciences, Tsinghua University, Beijing 100084, China.

E-mail address: hujiaxin@mail.tsinghua.edu.cn

Department of Mathematics, Chinese University of Hong Kong, Shatin, N.T., Hong Kong. E-mail address: kslau@math.cuhk.edu.hk 